OPEN ACCESS

Edited by:

Xiaojie Huang

Capital Medical University, China

Reviewed by:

Rolf Teschke,

Hospital Hanau, Germany

Andreas Benesic,

Krankenhaus GmbH Weilheim -

Schongau, Germany

${ }^{*}$ Correspondence:

Ying Wen

wenying666466@163.com

Qing Hai Hu

qinghaihucn@163.com

†These authors have contributed equally to this work

Specialty section:

This article was submitted to Infectious Diseases-Surveillance,

Prevention and Treatment,

a section of the journal

Frontiers in Medicine

Received: 18 November 2021

Accepted: 31 January 2022

Published: 22 February 2022

Citation:

Liu S, Zhou Y, Wang Y, Li CB, Wang W, Lu X, LiU P, Hu QH and Wen Y (2022) The Correlated Risk

Factors for Severe Liver Damage Among HIV-Positive Inpatients With Abnormal Liver Tests.

Front. Med. 9:817370.

doi: 10.3389/fmed.2022.817370

\section{The Correlated Risk Factors for Severe Liver Damage Among HIV-Positive Inpatients With Abnormal Liver Tests}

\author{
Sheng Liu ${ }^{1}$, Ying Zhou ${ }^{1}$, Yu Wang ${ }^{1}$, Cheng Bo Li ${ }^{1}$, Wen Wang ${ }^{1}$, Xu Lu ${ }^{1}$, Pei Liu ${ }^{1}$, \\ Qing Hai Hu ${ }^{2 \star t}$ and Ying Wen ${ }^{1 * t}$ \\ ${ }^{1}$ Infectious Diseases Department, The First Affiliated Hospital of China Medical University, Shenyang, China, ${ }^{2}$ Key Laboratory \\ of AIDS Immunology of Ministry of Health, Department of Laboratory Medicine, The First Affiliated Hospital of China Medical \\ University, Shenyang, China
}

Background: This study investigated the factors correlated with severe liver damage among HIV-infected inpatients.

Methods: We retrospectively collected the first hospitalized HIV-infected patients in the Department of Infectious Disease of the First Affiliated Hospital of China Medical University from January 1, 2010, to December 31, 2019. We used multivariate logistic regression to identify the factors associated with severe liver damage.

Results: A total of 493 patients with abnormal liver tests were recruited. Among 63 cases (12.8\%) with severe liver injury, drug-induced liver injury (DILI) identified by the updated Roussel Uclaf Causality Assessment Method (RUCAM) score as the direct cause was found in 43 cases. Anti-tuberculosis drug (ATD) exposure [adjusted odds ratio $(\mathrm{aOR})=1.835,95 \%$ confidence interval $(\mathrm{Cl}): 1.031-3.268]$, cotrimoxazole exposure ( $\mathrm{aOR}=2.775,95 \% \mathrm{Cl}: 1.511-5.096)$, comorbidity of viral hepatitis $(\mathrm{aOR}=2.340,95 \%$ $\mathrm{Cl}: 1.161-4.716)$, alcohol consumption history (aOR $=2.392,95 \% \mathrm{Cl}: 1.199-4.769)$, and thrombocytopenia $(\mathrm{aOR}=2.583,95 \% \mathrm{Cl}: 1.127-5.917)$ were associated with severe liver injury (all $P<0.05$ ).

Conclusions: DILI was the predominant cause of severe liver damage, followed by hepatitis virus co-infection. For patients with alcohol consumption and thrombocytopenia, frequent monitoring of liver function tests should be considered.

Keywords: acquired immune deficiency syndrome (AIDS), severe liver damage, antiretroviral therapy (ART), logistic regression model, risk factors

\section{BACKGROUND}

Liver-related death is the common cause of non-acquired immune deficiency syndrome (AIDS)-related death, which is mainly due to decompensated cirrhosis and hepatocellular carcinoma among human immunodeficiency virus (HIV) patients co-infected with hepatitis B virus (HBV) or hepatitis C virus (HCV) (1). Liver-related death is occasionally associated with fulminant hepatic failure (FHF) caused by drug-induced liver injury (DILI) and hepatitis virus co-infection $(2,3)$. In a cohort of antiretroviral therapy (ART)-experienced individuals from 
high-income countries, approximately $14.5 \%$ of deaths were from liver-related causes (4). Liver enzyme elevation is common among HIV-infected inpatients, and $50 \%$ of these patients are asymptomatic (5). The prevalence of mild and moderate liver enzyme elevations associated with steatosis/steatohepatitis among ART-treated patients was much higher than that among ART-naïve patients, mainly due to increased body mass index (BMI) (6-8). The liver damage incidence in China was highly observed within 6-12 months after ART initiation (9). DILI frequently occurs in patients with older ART regimens (10). Hazardous alcohol consumption is also a risk factor for liver disease in HIV-infected populations (11). The liver is also a commonly involved site due to common systemic opportunistic infections (OIs), including Mycobacterium tuberculosis (MTB), non-tuberculosis mycobacteria (NTM), fungi, and cytomegalovirus (CMV) (10). Furthermore, hepatic TB-immune reconstitution inflammatory syndrome (IRIS) was also an etiology of liver damage shortly after ART (10).

The occurrence of severe liver events often resulted in liverrelated hospital admissions, elevated risk of hepatic failure, and alteration in the medical care of other fatal illnesses. The alanine transaminase (ALT) was often the only index for monitoring liver disease in $\mathrm{HIV}$-positive populations $(12,13)$. At the same time, multiple variables were already used to evaluate liver injury (6). The alkaline phosphatase (ALP) and gamma-glutamyl transferase (GGT) should be assessed in cholestasis. The total bilirubin (TBIL), direct bilirubin (DBIL), cholinesterase (CHE), serum albumin (ALB), prothrombin time $(\mathrm{PT})$ and international normalized ratio (INR) should be assessed in liver failure or decompensated cirrhosis. To date, the correlated risk factors for severe liver events among inpatients are unknown. They may be much different from outpatients under long-term ART. Therefore, we carried out a retrospective study using multiple liver tests parameters to clarify the correlated risk factors of severe liver events in order to make rapid diagnoses and initiate prompt treatment.

\footnotetext{
Abbreviations: HBV, hepatitis B virus; HIV, human immunodeficiency virus; HCV, hepatitis C virus; AIDS, acquired immune deficiency syndrome; FHF, fulminant hepatic failure; DILI, drug-induced liver injury; ART, antiretroviral therapy; ALT, alanine transaminase; AST, aspartate aminotransferase; RUCAM, Roussel Uclaf Causality Assessment Method score; OIs, opportunistic infections; MTB, Mycobacterium tuberculosis; ATDs, anti-tuberculosis drugs; NTM, nontuberculosis mycobacteria; IRIS, immune reconstitution inflammatory syndrome; MSM, men who have sex with men; BMI, body mass index; CHE, cholinesterase; ALP, alkaline phosphatase; GGT, gamma-glutamyltransferase; TBIL, total bilirubin; DBIL, direct bilirubin; PT, prothrombin time; INR, international normalized ratio; ULN, upper limit of normality; WHO, World Health Organization; IQR, interquartile range; $\mathrm{OR}$, odds ratio; aOR, adjusted odds ratio; CI, confidence interval; HB, hemoglobin; ALB, albumin; Scr, serum creatinine; NSAIDs, non-steroidal anti-inflammatory drugs; TB, tuberculosis; TMP-SMZ, trimethoprim-sulfamethoxazole; NVP, nevirapine; EFV, efavirenz; CRP, C-reactive protein; PJP, pneumocystis jiroveci pneumonia; CNS, central nervous system; MAC, mycobacterium avium complex disease; ALD, alcohol-related liver disease; NAFLD, non-alcoholic fatty liver disease; CMV, cytomegalovirus; $\mathrm{HR}$, hazard ratio; APRI, the aspartate aminotransferase-to-platelet ratio index; FIB-4, the fibrosis index based on four factors; DRESS, eosinophilia and systemic symptoms; CLD, chronic liver disease.
}

\section{METHOD}

\section{Study Design and Patients Enrollment}

We conducted a retrospective study of inpatients who had already been diagnosed with HIV infection and were first admitted to the Department of Infectious Disease at the First Affiliated Hospital of China Medical University (Shenyang, China) from January 1, 2010, to December 31, 2019. Both cases with liver injury on admission and individuals without liver injury on admission but developing new liver injury during hospitalization were recruited. Exclusion criteria included: (1) patients with sustained normal liver function tests at admission and during the hospitalization; (2) patients whose ALT measurements at baseline were not available; (3) patients whose elevated ALP was due to skeletal injury; (4) patients whose elevated TBIL was due to hemolysis or extrahepatic bile duct obstructive diseases; and (5) patients with myositis. The Clinical Research Ethics Committee approved this study of the First Affiliated Hospital of China Medical University.

\section{Definitions of Outcomes and Covariates}

Clinical data, including demographic data, underlying medical conditions, liver tests and clinical course, were obtained from patients' medical records, which were accomplished by three authors simultaneously. We separately analyzed the factors that might influence severe liver damage.

Liver injuries were defined as participants who had at least one incident elevation of ALT, aspartate aminotransferase (AST), ALP, or TBIL above the upper limit of normality (ULN). Severe liver injury in our study was defined as individuals who had plasma ALT, AST, or ALP values > 5 times above the ULN or TBIL values $>3$ times above the ULN, which was according to at least grade 3 in commendations on management of immunemediated liver injury induced by immune checkpoint inhibitors (14); otherwise, they were defined as mild or moderate liver injury. Cholestasis was defined as individuals with ALP values $>1.25$ times the ULN and GGT > 3 times the ULN. Alcohol consumption was defined as patients who drank more than 40 grams (20 grams for female patients) of alcohol per day for more than 5 years (15). Cases of DILI were identified, assessed for a causality, categorized based on the updated Roussel Uclaf Causality Assessment Method (RUCAM) and DILI guidelines $(14,16,17)$ and the exclusion of other liver diseases. HCV infection was defined as individuals who were both HCVantibody positive and HCV-RNA positive. The resolved liver injury was defined as partial or complete restoration of the profile of liver tests when discharged compared to the worst profile during hospitalization. Hyponatremia was described as a serum sodium concentration $<135 \mathrm{mmol} / \mathrm{L}$. Hyperlipidemia was defined as serum triglycerides, total cholesterol, or lowdensity lipoprotein above the ULN (18). Thrombocytopenia was described as a platelet count $<100,000 / \mu \mathrm{L}$. The World Health Organization (WHO) clinical stage of all patients was based on the most severe clinical stage in their medical history records. Ultrasound was the diagnostic procedure for fatty liver. 


\section{Statistical Analysis}

The results were expressed as numbers, medians (interquartile ranges), and percentages. We compared conditions between patients with mild or moderate liver damage and patients with severe liver damage. The means for continuous variables were compared using the Student's $t$-test for normally distributed data. Otherwise, the Mann-Whitney U test was used. Proportions for categorical variables were compared using the chi-squared test. Fisher's exact test was used when the data were sparse. Univariate and multivariable logistic regression models were employed to assess factors associated with severe liver damage, with odds ratios (OR), adjusted odds ratios (aOR), and 95\% confidence intervals (CI). After assessing the $P$-value from the univariate model, variables with $P<0.1$ were introduced into multivariable logistic regression models. $P$-values $<0.05$ were considered to be statistically significant for all cases. All analyses were performed by using SPSS software for Windows version 22.0 (Chicago, IL).

\section{RESULT}

\section{Clinical Characteristics at Baseline}

Among 708 patients who had baseline liver tests, three patients were first excluded, including one patient with elevated ALP due to skeletal injury and two patients with myositis. Among the remaining 705 patients, 212 had sustained normal liver function tests at admission and during the hospitalization, while 493 had abnormal liver tests levels. The percentage of severe liver events among all inpatients was $8.9 \%$ (63/705). The rate of severe liver events among inpatients with abnormal liver tests was $12.8 \%(63 / 493)$.

A total of 493 patients with liver damage were enrolled in this study. A total of 441 cases $(89.5 \%)$ in our study were sexually transmitted, and 267 patients (54.2\%) were men who had sex with men (MSM). The median age was 36 years (range: 2079 years). There were three cases with compensated cirrhosis and six patients with decompensated cirrhosis. AIDS-related illnesses were still prominent problems for inpatients, including pneumocystis jirovecii pneumonia (PJP), TB, invasive fungal disease, malignancies, bacterial bloodstream infections, central nervous system (CNS) disease and disseminated mycobacterium avium complex (MAC) disease (Table 1). Among 493 patients with liver damage, a total of 49 patients died in the hospital. The in-hospital mortality rate was $9.9 \%$. Non-liver-related death accounted for $98.0 \%$ of deaths. The direct causes of death included PJP (20), TB (16), sepsis syndrome (3), malignant tumors (2), cryptococcal meningitis (3), invasive pulmonary aspergillosis infection (3), HIV encephalopathy (1) and hepatic failure (1). The liver function tests levels of patients with severe liver injury compared with mild or moderate-liver injury patients were presented in Figure 1. Other common laboratory test abnormalities were summarized in Table $\mathbf{1}$.

Compared with the patients with mild or moderate liver damage, the patients with severe liver damage were more likely to have anti-tuberculosis drug (ATD) application (49.2 vs. $32.8 \%$, $p=0.037$ ), cotrimoxazole exposure (65.1 vs. $46.5 \%, p=0.010$ ), viral hepatitis (26.9 vs. $11.8 \%, p<0.001)$, a history of alcohol
TABLE 1 | Baseline clinical and laboratory characteristics of 493 hospitalized HIV patients.

\begin{tabular}{|c|c|}
\hline Data & No. (\%) \\
\hline Age (years) $\leq 40$ & $291(59.0 \%)$ \\
\hline$>40$ & $202(41.0 \%)$ \\
\hline Male & 465 (94.3\%) \\
\hline $\mathrm{BMl}<18.5 \mathrm{~kg} / \mathrm{m}^{2}$ & $383(77.7 \%)$ \\
\hline Alcohol consumption history & $68(13.8 \%)$ \\
\hline Hyperlipemia & $110(22.3 \%)$ \\
\hline Diabetes & $26(5.3 \%)$ \\
\hline Hypertension & $17(3.4 \%)$ \\
\hline ART prior to admission & $236(47.9 \%)$ \\
\hline NSAID exposure $>7$ days before admission & $309(62.7 \%)$ \\
\hline Cotrimoxazole exposure & $241(48.9 \%)$ \\
\hline ATDs exposure & $213(43.2 \%)$ \\
\hline Anti- fungal drugs exposure & $31(6.3 \%)$ \\
\hline HBV coinfection & $53(10.8 \%)$ \\
\hline HCV coinfection & $15(3.0 \%)$ \\
\hline NAFLD & $21(4.3 \%)$ \\
\hline ALD & $17(3.4 \%)$ \\
\hline WHO clinical stage III-IV & $434(88.0 \%)$ \\
\hline PJP & $241(48.9 \%)$ \\
\hline TB & $172(34.9 \%)$ \\
\hline CNS infection & $66(13.4 \%)$ \\
\hline Malignant tumor & $33(6.6 \%)$ \\
\hline Invasive fungal infection & $31(6.3 \%)$ \\
\hline Bacterial bloodstream infection & $29(5.9 \%)$ \\
\hline Cytomegalovirus retinitis & $38(5.3 \%)$ \\
\hline Disseminated Mycobacterium avium complex disease & $9(1.9 \%)$ \\
\hline CD4 T counts < $200(/ \mu \mathrm{L})$ & $403(81.7 \%)$ \\
\hline $\mathrm{CRP}(>10 \mathrm{mg} / \mathrm{L})$ & $356(72.2 \%)$ \\
\hline Albumin $(<30 \mathrm{~g} / \mathrm{L})$ & $218(44.2 \%)$ \\
\hline $\mathrm{PT}>13.7 \mathrm{~s}$ & $205(41.6 \%)$ \\
\hline Hyponatremia & $197(40.0 \%)$ \\
\hline $\mathrm{HB}(<9 \mathrm{~g} / \mathrm{L})$ & $58(11.8 \%)$ \\
\hline Thrombocytopenia & $48(9.7 \%)$ \\
\hline $\operatorname{Scr}(>104 \mu \mathrm{mol} / \mathrm{L})$ & $5(1.0 \%)$ \\
\hline
\end{tabular}

ART, antiretroviral therapy; BMI, body mass index; ATDs, anti-tuberculosis drugs; $H B V$, hepatitis B virus; HCV, hepatitis C virus; ALD, alcoholic liver disease; NAFLD, nonalcoholic fatty liver disease; PJP, pneumocystis jiroveci pneumonia; TB, tuberculosis; CNS, central nervous system; HIV, human immunodeficiency virus; CMV, cytomegalovirus; CRP, C-reactive protein; ALT, glutamic alanine transaminase; $H B$, hemoglobin; SCr, serum creatinine.

consumption ( 25.4 vs. $12.1 \%, p=0.004)$ and thrombocytopenia (20.6 vs. $8.1 \%, p=0.002$ ) (Table 2 ).

\section{Treatment Adjustment After Severe Liver Damage}

Previous drugs should be modified when severe liver damage occurs. Drugs with hepatotoxicity should be avoided. Liver protective drugs, such as silymarin capsules, ursodeoxycholic acid capsules, bicyclol tablets, isoglycyrrhizinate magnesium injections, S-adenosylmethionine injections and L-glutathione injections are usually selectively used. 

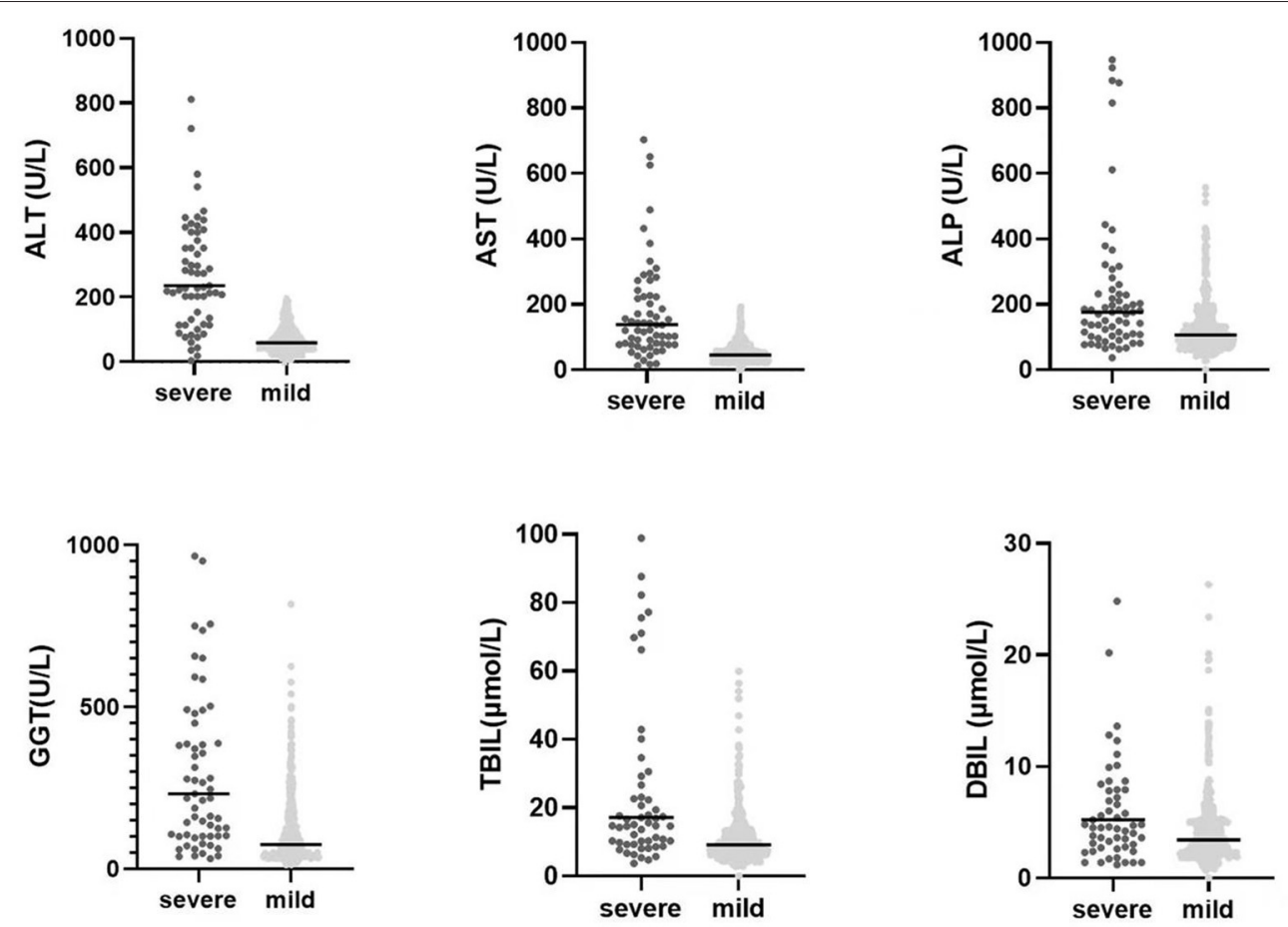

FIGURE 1 | The comparision of liver function tests levels between mild or moderate liver injury HIV-infected inpatients and severe liver injury HIV-infected inpatients. The $P$-values of all of six parameters were $<0.05$ with statistically significant by using the Mann Whitney $U$ test.

\section{Factors Associated With Severe Liver Injury}

Among 493 patients with abnormal liver parameters, 213 cases presented with liver injury at the baseline visit. At the same time, 280 cases developed new liver damage during hospitalization. Resolved liver injury was observed in $100 \%$ of 430 cases with mild or moderate grades of liver injury. Among 63 patients with severe liver injury, 32 cases met the definition of severe liver injury on admission, and 31 cases developed severe liver injury during hospitalization. Twenty-six patients with severe liver injury met the criteria of cholestasis. Among patients with severe liver injury, 51 cases $(81.0 \%)$ resolved when discharged. Among 12 cases with unresolved or worsened liver injury, one patient died in the hospital due to hepatic failure.

The direct causes of severe liver injury in 63 cases included DILI (43), viral hepatitis (12), sepsis syndrome (4), CMV (2), EBV (1), and malignant tumor (1). Six patients met the criteria of liver failure, including subacute liver failure (5) and chronic liver failure (1). Among cases with severe liver injuries, DILI was identified and scored in $68.3 \%$ (43/63) patients including 3 cases with high probable DILI, 36 cases with probable DILI, 4 cases with possible DILI. The pattern of DILI was hepatocellular in $36.5 \%(23 / 43)$ of patients, cholestatic in $25.6 \%(11 / 43)$ of patients, and mixed in $20.9 \%(9 / 43)$ of patients. Nine patients with DILI had fever and eruptions. One patient with nevirapine (NVP)-DILI presented with rash with eosinophilia and systemic symptoms (DRESS). Cases with DILI were caused by ATDs (11), cotrimoxazole (10), NVP (10), other antibiotics (5), efavirenz (4), and non-steroidal anti-inflammatory drugs (NSAIDs) (3).
Hepatitis virus co-infection accounted for $19.0 \%$ (12/63) of cases with severe liver injury, while five cases met the criteria for IRIS. Among six patients with cirrhosis in severe liver damage group, there were 5 cases at Child-Pugh class B and one case at ChildPugh class C. Only one patient was aware of his HBV-infected status prior to HIV diagnosis. Among 63 patients with severe liver injury, seven patients died in the hospital, including six patients with non-hepatic-associated death and one patient with hepatic-associated death.

Data with a $P$-value $<0.1$ in the univariate analysis were entered into the multivariate logistic regression model, including exposure to ATD $(P=0.012)$, cotrimoxazole exposure $(P=$ $0.007)$, viral hepatitis $(P=0.004)$, alcohol consumption history $(P=0.005)$, and thrombocytopenia $(P=0.007)$. Finally, analyses using the multivariate logistic regression model showed that exposure to ATD $(\mathrm{aOR}=1.835,95 \% \mathrm{CI}: 1.031-3.268 ; P=0.039)$, cotrimoxazole exposure $(\mathrm{aOR}=2.775,95 \% \mathrm{CI}: 1.511-5.096 ; P$ $=0.001$ ), comorbidity of viral hepatitis $(\mathrm{aOR}=2.340,95 \% \mathrm{CI}$ : 1.161-4.716; $P=0.017$ ), alcohol consumption history $(\mathrm{aOR}=$ 2.392, 95\% CI: $1.199-4.769 ; P=0.013)$ and thrombocytopenia $(\mathrm{aOR}=2.583,95 \% \mathrm{CI}: 1.127-5.917 ; P=0.025)$ were risk factors for severe liver injury (Table 3 ).

\section{DISCUSSION}

In this study, the percentage of severe liver events among all inpatients was $8.9 \%$, much higher than the $0.3 \%$ of ARTnaïve studies (12). Among cases with severe liver damage, DILI was the predominant finding $(68.3 \%)$, followed by hepatitis 
TABLE 2 | Baseline characteristics of patients with severe liver injury compared with mild or moderate-liver injury patients.

\begin{tabular}{|c|c|c|c|}
\hline Characteristics & $\begin{array}{l}\text { Severe liver } \\
\text { injury }[n(\%)]\end{array}$ & $\begin{array}{c}\text { Mild or } \\
\text { moderate liver } \\
\text { injury [n (\%)] }\end{array}$ & $\boldsymbol{P}$ \\
\hline Total & $63(100)$ & $430(100)$ & \\
\hline Age, years [median (IQR)] & $37(32,50)$ & $36(29,48)$ & 0.267 \\
\hline Age $>40$ years & $29(46.0)$ & $173(40.2)$ & 0.382 \\
\hline Male & $61(96.8)$ & $404(93.9)$ & 0.358 \\
\hline $\mathrm{BMI}\left(\mathrm{kg} / \mathrm{m}^{2}\right)$ & & & 0.615 \\
\hline$<18.5$ & $50(79.4)$ & $333(77.4)$ & \\
\hline $18.5-23.9$ & $2(3.2)$ & $27(3.2)$ & \\
\hline$\geq 24$ & $11(17.5)$ & 70 (16.3) & \\
\hline Underlying medical condition & $14(22.2)$ & $58(13.5)$ & 0.067 \\
\hline WHO clinical stage III-IV & $53(84.1)$ & 381 (88.6) & 0.307 \\
\hline Alcohol consumption history & $16(25.4)$ & $52(12.1)$ & $0.004^{\#}$ \\
\hline ART prior to admission & $34(53.9)$ & $202(46.9)$ & 0.300 \\
\hline ART regimen & & & 0.752 \\
\hline NVP-containing ART regimen & $22(34.9)$ & 137 (31.9) & \\
\hline Non-NVP-containing ART regimen & $23(36.5)$ & $150(34.9)$ & \\
\hline Without ART & $18(28.6)$ & $143(33.3)$ & \\
\hline Hospital stay days [median (IQR)] & $14(10,20)$ & $13(8,18)$ & 0.227 \\
\hline Hospital mortality & $8(12.7)$ & $41(9.5)$ & 0.433 \\
\hline CD4 < $200(/ \mu \mathrm{L})$ & $55(87.3)$ & $348(80.9)$ & 0.222 \\
\hline Hyperlipidemia & $16(25.4)$ & $94(21.8)$ & 0.529 \\
\hline Viral hepatitis & $17(26.9)$ & $51(11.8)$ & $<0.001^{\#}$ \\
\hline ALD or NAFLD & $5(7.9)$ & $33(7.6)$ & 0.942 \\
\hline Thrombocytopenia & $13(20.6)$ & $35(8.1)$ & $0.002^{\#}$ \\
\hline Hyponatremia & $20(31.7)$ & $177(41.2)$ & 0.154 \\
\hline $\mathrm{CRP}>10 \mathrm{mg} / \mathrm{L}$ & $40(63.4)$ & $316(73.5)$ & 0.098 \\
\hline Cotrimoxazole exposure & $41(65.1)$ & 200 (46.5) & $0.010^{\#}$ \\
\hline Cotrimoxazole before admission & $10(15.8)$ & $34(7.9)$ & \\
\hline Cotrimoxazole after admission & $31(49.2)$ & $166(38.6)$ & \\
\hline ATDs exposure & $35(49.2)$ & $178(32.8)$ & $0.037^{\#}$ \\
\hline ATDs before admission & $13(20.6)$ & 57 (13.3) & \\
\hline ATDs after admission & $22(34.9)$ & $121(28.1)$ & \\
\hline Anti- fungal drugs exposure & $6(9.5)$ & $25(5.8)$ & 0.258 \\
\hline $\begin{array}{l}\text { NSAID exposure }>7 \text { days before } \\
\text { admission }\end{array}$ & 38 (60.3) & $271(63.0)$ & 0.679 \\
\hline $\mathrm{HB}<9 \mathrm{~g} / \mathrm{dL}$ & $5(8.0)$ & $53(12.3)$ & 0.783 \\
\hline ALB $<30 \mathrm{~g} / \mathrm{L}$ & $28(44.4)$ & $190(44.2)$ & 0.969 \\
\hline $\mathrm{PT}>13.7 \mathrm{~s}$ & 27 (42.9) & 178 (41.4) & 0.826 \\
\hline $\mathrm{Scr}>104 \mathrm{mmol} / \mathrm{L}$ & $1(1.6)$ & $4(0.9)$ & 0.627 \\
\hline $\mathrm{CHE}<4,000(\mathrm{U} / \mathrm{L})$ & $13(20.6)$ & $99(23.0)$ & 0.673 \\
\hline
\end{tabular}

${ }^{\#} P$-value $<0.05$, statistically significant with the use of chi-square test or Fisher's exact test.

IQR, interquartile range; BMI, body mass index; WHO, World Health Organization; ART, anti-retroviral therapy; NVP, nevirapine; ALD, alcohol-related liver disease; NAFLD, nonalcoholic fatty liver disease; CRP, C-reactive protein; ATDs, anti-tuberculosis drugs; NSAIDs, non-steroidal anti-inflammatory drugs; HB, hemoglobin; ALB, albumin; PT, prothrombin time; Scr, serum creatinine; CHE, cholinesterase.

virus co-infection (19.0\%). PJP and TB were two main coexistent OIs among inpatients. The post-exposure monitoring of cotrimoxazole and ATD should be recommended. The presence of thrombocytopenia was a high-risk factor for the occurrence of severe liver injury. Although most liver tests
TABLE 3 | Factors associated with severe liver injury among HIV-infected inpatients in Shenyang.

\begin{tabular}{|c|c|c|c|c|}
\hline Factors & OR $(95 \% \mathrm{Cl})$ & P1 & Adjust OR (95\%Cl) & $\mathbf{P 2}$ \\
\hline \multicolumn{5}{|c|}{ Viral hepatitis } \\
\hline No & 1.000 & & & \\
\hline Yes & $2.530(1.336-4.789)$ & 0.004 & $2.340(1.161-4.716)$ & 0.017 \\
\hline \multicolumn{5}{|c|}{ Cotrimoxazole exposure } \\
\hline No & 1.000 & & & \\
\hline Yes & $2.143(1.235-3.720)$ & 0.007 & $2.775(1.511-5.096)$ & 0.001 \\
\hline \multicolumn{5}{|c|}{ Anti-TB drugs exposure } \\
\hline No & 1.000 & & & \\
\hline Yes & $1.986(1.165-3.385)$ & 0.012 & $1.835(1.031-3.268)$ & 0.039 \\
\hline \multicolumn{5}{|c|}{ Thrombocytopenia } \\
\hline No & 1.000 & & & \\
\hline Yes & $2.709(1.320-5.557)$ & 0.007 & $2.583(1.127-5.917)$ & 0.025 \\
\hline \multicolumn{5}{|c|}{ Alcohol consumption history } \\
\hline No & 1.000 & & & \\
\hline Yes & $2.475(1.309-4.679)$ & 0.005 & $2.392(1.199-4.769)$ & 0.013 \\
\hline
\end{tabular}

abnormalities resolved over time, they were not completely reversible in a small number of cases.

The acute DILI in our study occurred mainly within the first 3 months after taking drugs. The early-onset (within 12 weeks) DILI with a rash or fever were associated with hypersensitivity reactions, which showed a higher prevalence in HIV-infected individuals with advanced immunodeficiency than in the general population $(19,20)$. Non-nucleoside reverse transcriptase inhibitors, such as NVP and efavirenz (EFV), showed a high possibility of liver injury $(2,21)$. NVP was associated with non-specific hepatitis, while EFV was associated with submassive necrosis (7). Compared to EFV, NVP showed more frequent severe hepatic injury in our study. However, NVP-containing ART regimens were not a significant risk factor for severe liver damage due to its usual presentation of mild to moderate liver injury (22) and genetic polymorphisms (23). Luckily, new medications without apparent hepatotoxicity have become widely available. ATD were an important cause of DILI because one-third of patients had TB co-infection in our study. There were high incidence rates of DILI among cotreated HIV/TB co-infected patients (24). Although liver tests abnormalities were generally reversible within 4-6 weeks of discontinuation of the offending drugs, DILI caused by ATD was associated with high mortality (25). Hepatotoxicity was also associated with isoniazid preventive therapy (26). Among antibiotic-induced liver injuries, cotrimoxazole has the highest risk of cholestatic or ductopenic injury $(7,27)$. Approximately half of the inpatients were diagnosed with PJP in this study. Although NSAIDs are available and widely used for antipyretics in China, they are not associated with increased liver damage risk due to the lack of overdoses. The application of traditional Chinese medicine or herbal and dietary supplements is uncommon in the HIV-positive population. Apart from DILI, the updated RUCAM was also recommended for assessing herbinduced liver injury $(28,29)$. 
Patients co-infected with $\mathrm{HBV}$ or $\mathrm{HCV}$ were prone to liver injury, identical to a previous study (30). HCV co-infection presented a high risk of developing severe liver toxicity related to NVP (31). An approximately $10.8 \%$ prevalence of HBV coinfection was found in our study. In comparison, the prevalence of HCV co-infection was only $3.0 \%$. HBV co-infection was a more common factor associated with severe liver injury than $\mathrm{HCV}$ co-infection in our study. Most patients were unaware of their HBV-infected status at the time of HIV diagnosis. HBeAgnegative chronic HBV infection with a high HBV DNA load is an apparent characteristic (32). Hepatic flares were occasionally considered as hepatitis virus-associated IRIS (33). Compared to intravenous drug users and former plasma donors, most cases were sexually transmitted in our study, who might be less associated with HCV infection.

Our study indicated the importance of addressing previous alcohol use among inpatients, a high-risk population for severe liver damage. Patients with binge drinking were more often to experience a liver-related event, even liver-related death (34). Hazardous drinking is a significant risk factor for liver fibrosis, particularly among HIV-positive patients without HCV coinfection (35). The underlying mechanism is that alcohol metabolism potentiates HIV-induced hepatotoxicity (36). Notably, alcohol intake $>40 \mathrm{~g}$ per day was associated with severe liver toxicity in those patients receiving NVP-or EFV-containing regimens (37).

Thrombocytopenia was also a contributing factor for the occurrence of severe liver damage in this study. Thrombocytopenia is common in HIV-infected patients. Its seriousness and incidence are related to the stage of HIV infection, hypersplenism, portal hypertension and other mechanisms (38). As the common tools for assessing chronic liver disease (CLD), both aspartate aminotransferase-to-platelet ratio index (APRI) and fibrosis index based on four factors (FIB4) could be affected by platelet counts. Therefore, evaluation for liver fibrosis should be performed among cases with thrombocytopenia, which would be a better combination with transient elastography.

Elevated ALP, GGT, and TBIL were common in cases with IRIS-associated liver injury (39). Liver injury associated with TBIRIS is characterized by the infiltration of neutrophils, plasma cells, and abundant lymphocytes within granulomas (40). Liver biopsy of cases with HBV-IRIS showed lymphocytic infiltration, predominantly diffuse CD8 $+\mathrm{T}$ cell infiltration in the portal areas and lobules (41). Elevated TBIL was more common than elevated ALT in patients with decompensated liver cirrhosis or liver abscesses. We should note that most severe liver events in this study were associated with acute reversible liver damage in the absence of pre-existing CLD. Low serum ALB levels are prevalent laboratory test abnormalities in HIV-infected patients, irrespective of liver injury. Prolonged PT was sensitive but nonspecific to acute or chronic liver failure. Abdominal ultrasound examination or computed tomography scans were recommended for routine screening among inpatients in our department. Although clinicopathological diagnosis is essential, liver biopsy is not always necessary in patients with acute reversible liver injury.
Our study is associated with some limitations. Only hospitalized patients were included in this analysis, and they lacked liver biopsies and follow-up studies after discharge. Inpatients outside the Department of Infectious Disease in our hospital were excluded in this retrospective study because there was a lack of intensified medical care strategy and professional assessment of HIV inpatients in other departments. Importantly, we could collect detailed information on potentially hepatotoxic medications and frequently monitor liver conditions using multiple variables among inpatients. Our data are only from our hospital, and further studies from multiple centers should be conducted and verified.

In conclusion, the severe liver injury event is not uncommon among HIV-infected inpatients. Patients with HBV co-infection and cotrimoxazole or ATD-induced DILI deserve special medical care and frequent monitoring of liver tests. Among patients with a history of alcohol consumption and thrombocytopenia, we should further assess liver fibrosis. These findings have important implications for identifying risky individuals who are more likely to develop severe liver injury and improve therapeutic regimens.

\section{DATA AVAILABILITY STATEMENT}

The raw data supporting the conclusions of this article will be made available by the authors, without undue reservation.

\section{ETHICS STATEMENT}

The studies involving human participants were reviewed and approved by the Clinical Research Ethics Committee of the First Affiliated Hospital of China Medical University. The patients/participants provided their written informed consent to participate in this study.

\section{AUTHOR CONTRIBUTIONS}

SL was responsible for data collection, statistic analysis, and article writing. YZ, YWa, CL, WW, XL, and PL participated in the clinical diagnosis and treatment. YWe designed the article and took part in writing and revising. $\mathrm{QH}$ designed the study and was responsible for the statistical analysis. All authors contributed to the article and approved the submitted version.

\section{FUNDING}

This work was supported by the Double First-Class University and Discipline Construction funds of China Medical University (3110119068 to YWe) and the Fund of National Natural Science (82073620 to QH). The funders had no role in the study design, data collection, and analysis.

\section{ACKNOWLEDGMENTS}

The authors extend their sincere thanks to all participants in the study. The authors also thank all colleagues in the infectious disease of the First Affiliated Hospital of China Medical University for their professional assistance. 


\section{REFERENCES}

1. Rosenthal E, Roussillon C, Salmon-Ceron D, Georget A, Henard S, Huleux T, et al. Liver-related deaths in Hiv-infected patients between 1995 and 2010 in France: the mortavic 2010 study in collaboration with the Agence Nationale De Recherche Sur Le Sida (Anrs) En 20 Mortalite 2010 Survey. HIV Med. (2015) 16:230-9. doi: 10.1111/hiv.12204

2. Bhattacharya D, Gupta A, Tierney C, Huang S, Peters MG, Chipato T, et al. Hepatotoxicity and liver-related mortality in women of childbearing potential living with human immunodeficiency virus and high $\mathrm{Cd} 4$ cell counts initiating Efavirenz-containing regimens. Clin Infect Dis. (2021) 72:1342-9. doi: $10.1093 /$ cid/ciaa244

3. Kramer JR, Giordano TP, Souchek J, El-Serag HB. Hepatitis C coinfection increases the risk of fulminant hepatic failure in patients with Hiv in the haart era. J Hepatol. (2005) 42:309-14. doi: 10.1016/j.jhep.2004.11.017

4. Weber R, Sabin CA, Friis-Moller N, Reiss P, El-Sadr WM, Kirk $\mathrm{O}$, et al. Liver-related deaths in persons infected with the human immunodeficiency virus: the D:A:D study. Arch Intern Med. (2006) 166:1632-41. doi: 10.1001/archinte.166.15.1632

5. Joshi D, O'Grady J, Dieterich D, Gazzard B, Agarwal K. Increasing burden of liver disease in patients with hiv infection. Lancet. (2011) 377:1198-209. doi: 10.1016/S0140-6736 (10)62001-6

6. Tesfa E, Siefu D, Belayneh Y, Mekonnen Z. Liver enzyme elevation in patients taking haart compared with treatment naive controls at Debre Berhan referral hospital: a comparative cross-sectional study, Northeast Ethiopia. BMC Res Notes. (2019) 12:714. doi: 10.1186/s13104-019-4748-4

7. Sonderup MW, Wainwright H, Hall P, Hairwadzi H, Spearman CW. A clinicopathological cohort study of liver pathology in 301 patients with human immunodeficiency virus/acquired immune deficiency syndrome. Hepatology. (2015) 61:1721-9. doi: 10.1002/hep.27710

8. Iogna Prat L, Roccarina D, Lever R, Lombardi R, Rodger A, Hall A, et al. Etiology and severity of liver disease in Hiv-positive patients with suspected Nafld: lessons from a cohort with available liver biopsies. J Acquir Immune Defic Syndr. (2019) 80:474-80. doi: 10.1097/QAI.00000000000 01942

9. Qin F, Jiang J, Qin C, Huang Y, Liang B, Xu Y, et al. Liver damage in patients living with Hiv on antiretroviral treatment with normal baseline liver function and without $\mathrm{Hbv} / \mathrm{Hcv}$ infection: an 11-year retrospective cohort study in Guangxi, China. BMJ Open. (2019) 9:e023140. doi: 10.1136/bmjopen-2018-023140

10. Acharya C, Dharel N, Sterling RK. Chronic liver disease in the human immunodeficiency virus patient. Clin Liver Dis. (2015) 19:1-22. doi: 10.1016/j.cld.2014.09.001

11. Shanyinde M, Girardi E, Puoti M, De Luca A, Sighinolfi L, Caterina UF, et al. Is physician assessment of alcohol consumption useful in predicting risk of severe liver disease among people with Hiv and HIV/HCV co-infection? BMC Public Health. (2019) 19:1291. doi: 10.1186/s12889-019-7608-1

12. Nagu TJ, Kanyangarara M, Hawkins C, Hertmark E, Chalamila G, Spiegelman $D$, et al. Elevated alanine aminotransferase in antiretroviral-naive Hiv-infected african patients: magnitude and risk factors. HIV Med. (2012) 13:541-8. doi: 10.1111/j.1468-1293.2012.01006.x

13. Mugusi SF, Sando D, Mugusi FM, Hawkins C, Aboud S, Fawzi WW, et al. Risk factors for alanine aminotransferase elevations in a prospective cohort of Hiv-infected tanzanian adults initiating antiretroviral therapy. J Int Assoc Provid AIDS Care. (2019) 18:2325958219884939. doi: 10.1177/23259582198 84939

14. Easl clinical practice guidelines: drug-induced liver injury. J Hepatol. (2019) 70:1222-61. doi: 10.1016/j.jhep.2019.02.014

15. Guidelines of prevention and treatment for alcoholic liver disease: a 2018 update. Zhonghua Gan Zang Bing Za Zhi. (2018) 26:188-94. doi: $10.3760 / \mathrm{cma}$.j.issn.1007-3418.2018.03.007

16. Danan G, Teschke R. Rucam in drug and herb induced liver injury: the update. Int J Mol Sci. (2015) 17:14. doi: 10.3390/ijms17010014

17. Devarbhavi H, Aithal G, Treeprasertsuk S, Takikawa H, Mao Y, Shasthry SM, et al. Drug-induced liver injury: asia pacific association of study of liver consensus guidelines. Hepatol Int. (2021) 15:258-82. doi: 10.1007/s12072-021-10144-3
18. Chinese guidelines on prevention and treatment of dyslipidemia in adults. Zhonghua Xin Xue Guan Bing Za Zhi. (2007) 35:390-419. doi: 10.3760/j.issn:0253-3758.2007.05.003

19. Johnson JR. Cutaneous disease and drug reactions in HIV infection. $N$ Engl J Med. (1993) 329:1582. doi: 10.1056/NEJM199311183292119

20. Hoosen K, Mosam A, Dlova NC, Grayson W. An update on adverse cutaneous drug reactions in HIV/AIDS. Dermatopathology. (2019) 6:111-25. doi: 10.1159/000496389

21. Nunez M. Hepatotoxicity of antiretrovirals: incidence, mechanisms and management. J Hepatol. (2006) 44 (1 Suppl.):S132-9. doi: 10.1016/j.jhep.2005.11.027

22. Li ZC, Li HJ, Dai LL, Gao YQ, Cai WP, Li HY, et al. Liver injury in Hiv-1-infected patients receiving non-nucleosides reverse transcriptase inhibitors-based antiretroviral therapy. Chin Med J. (2010) 123:3587-90. doi: 10.1016/S1201-9712 (09)60541-5

23. Cornejo Castro EM, Carr DF, Jorgensen AL, Alfirevic A, Pirmohamed M. Hla-allelotype associations with nevirapine-induced hypersensitivity reactions and hepatotoxicity: a systematic review of the literature and meta-analysis. Pharmacogenet Genomics. (2015) 25:186-98. doi: 10.1097/FPC.0000000000000124

24. Naidoo K, Hassan-Moosa R, Mlotshwa P, Yende-Zuma N, Govender D, Padayatchi N, et al. High rates of drug-induced liver injury in people living with Hiv coinfected with tuberculosis $(\mathrm{Tb})$ irrespective of antiretroviral therapy timing during antituberculosis treatment: results from the starting antiretroviral therapy at three points in Tb trial. Clin Infect Dis. (2020) 70:2675-82. doi: $10.1093 / \mathrm{cid} / \mathrm{ciz} 732$

25. Abid A, Subhani F, Kayani F, Awan S, Abid S. Drug induced liver injury is associated with high mortality-a study from a tertiary care hospital in Pakistan. PLoS ONE. (2020) 15:e0231398. doi: 10.1371/journal.pone.02 31398

26. Ngongondo M, Miyahara S, Hughes MD, Sun X, Bisson GP, Gupta A, et al. Hepatotoxicity during isoniazid preventive therapy and antiretroviral therapy in people living with Hiv with severe immunosuppression: a secondary analysis of a multi-country open-label randomized controlled clinical trial. J Acquir Immune Defic Syndr. (2018) 78:54-61. doi: 10.1097/QAI.0000000000001641

27. Ferrajolo C, Verhamme KM, Trifiro G, t Jong GW, Picelli G, Giaquinto $\mathrm{C}$, et al. Antibiotic-induced liver injury in paediatric outpatients: a casecontrol study in primary care databases. Drug Saf. (2017) 40:305-15. doi: $10.1007 /$ s40264-016-0493-y

28. Zhu Y, Niu M, Wang JB, Wang RL, Li JY, Ma YQ, et al. Predictors of poor outcomes in 488 patients with herb-induced liver injury. Turk J Gastroenterol. (2019) 30:47-58. doi: 10.5152/tjg.2018.17847

29. Teschke R, Eickhoff A, Schulze J, Danan G. Herb-induced liver injury (Hili) with 12,068 worldwide cases published with causality assessments by roussel Uclaf causality assessment method (Rucam): an overview. Transl Gastroenterol Hepatol. (2021) 6:51. doi: 10.21037/tgh-20-149

30. Lana R, Nunez M, Mendoza JL, Soriano V. Rate and risk factors of liver toxicity in patients receiving antiretroviral therapy. Med Clin. (2001) 117:60710. doi: 10.1016/S0025-7753 (01)72194-X

31. Giacomelli A, Riva A, Falvella FS, Oreni ML, Cattaneo D, Cheli S, et al. Clinical and genetic factors associated with increased risk of severe liver toxicity in a monocentric cohort of Hiv positive patients receiving nevirapine-based antiretroviral therapy. BMC Infect Dis. (2018) 18:556. doi: 10.1186/s12879-018-3462-5

32. Demosthenes JP, Sachithanandham J, Fletcher GJ, Zachariah UG, Varghese GM, John Daniel HD, et al. Characteristics of treatment-naive Hbv-infected individuals with Hiv-1 coinfection: a cross-sectional study from South India. Indian J Med Microbiol. (2019) 37:219-24. doi: 10.4103/ijmm.IJMM_19_16

33. Yoshikawa S, Yoshio S, Yoshida Y, Tsutsui Y, Kawai H, Yamazoe T, et al. Impact of immune reconstitution-induced hepatic flare on hepatitis B surface antigen loss in hepatitis B virus/human immunodeficiency virus-1 coinfected patients. J Infect Dis. (2021) 223:2080-9. doi: 10.1093/infdis/jiaa662

34. Surial B, Bertholet N, Daeppen JB, Darling KEA, Calmy A, Gunthard HF, et al. The impact of binge drinking on mortality and liver disease in the Swiss Hiv cohort study. J Clin Med. (2021) 10:295. doi: 10.3390/jcm100 20295 
35. Chaudhry AA, Sulkowski MS, Chander G, Moore RD. Hazardous drinking is associated with an elevated aspartate aminotransferase to platelet ratio index in an urban Hiv-infected clinical cohort. HIV Med. (2009) 10:133-42. doi: 10.1111/j.1468-1293.2008.00662.x

36. Ganesan M, New-Aaron M, Dagur RS, Makarov E, Wang W, Kharbanda $\mathrm{KK}$, et al. Alcohol metabolism potentiates Hiv-induced hepatotoxicity: contribution to end-stage liver disease. Biomolecules. (2019) 9:851. doi: 10.3390/biom9120851

37. Ena J, Amador C, Benito C, Fenoll V, Pasquau F. Risk and determinants of developing severe liver toxicity during therapy with nevirapine-and efavirenz-containing regimens in Hiv-infected patients. Int J STD AIDS. (2003) 14:776-81. doi: 10.1258/09564620360 719840

38. Torre D, Pugliese A. Platelets and Hiv-1 infection: old and new aspects. Curr HIV Res. (2008) 6:411-8. doi: 10.2174/157016208785861140

39. Verma S, Bhakta H, Nowain A, Pais S, Kanel G, Squires K. Severe cholestatic liver injury days after initiating antiretroviral therapy in a patient with aids: drug toxicity or immune reconstitution inflammatory syndrome? Dig Dis Sci. (2005) 50:1813-7. doi: 10.1007/s10620-0052943-8

40. Barr DA, Ramdial PK. Clinicopathological correlates in Hiv seropositive tuberculosis cases presenting with jaundice after initiating antiretroviral therapy with a structured review of the literature. BMC Infect Dis. (2012) 12:257. doi: 10.1186/1471-2334-12-257
41. Mitsumoto F, Murata M, Kato Y, Ura K, Takayama K, Hiramine $\mathrm{S}$, et al. Hepatitis $\mathrm{B}$ virus-related immune reconstitution inflammatory syndrome in two patients coinfected with human immunodeficiency virus diagnosed with a liver biopsy. Intern Med. (2014) 53:2165-70. doi: $10.2169 /$ internalmedicine.53.2503

Conflict of Interest: The authors declare that the research was conducted in the absence of any commercial or financial relationships that could be construed as a potential conflict of interest.

Publisher's Note: All claims expressed in this article are solely those of the authors and do not necessarily represent those of their affiliated organizations, or those of the publisher, the editors and the reviewers. Any product that may be evaluated in this article, or claim that may be made by its manufacturer, is not guaranteed or endorsed by the publisher.

Copyright (c) $2022 \mathrm{Liu}, \mathrm{Zhou}, \mathrm{Wang}, \mathrm{Li}$, Wang, Lu, Liu, Hu and Wen. This is an open-access article distributed under the terms of the Creative Commons Attribution License (CC BY). The use, distribution or reproduction in other forums is permitted, provided the original author(s) and the copyright owner(s) are credited and that the original publication in this journal is cited, in accordance with accepted academic practice. No use, distribution or reproduction is permitted which does not comply with these terms. 